\section{Determinantes de la equidad en el financiamiento de los medicamentos en Argentina: un estudio empírico}

\author{
Determinants of equity in financing medicines in \\ Argentina: an empirical study
}

\section{Determinantes da equidade no financiamento de medicamentos na Argentina: um estudo empírico}

Mariana Dondo 1,2

Mauricio Monsalvo 2,3

Lucas A. Garibaldi 1,2

\title{
Resumen
}

Los medicamentos constituyen un alto porcentaje del gasto en salud de los hogares, por eso, tener un sistema progresivo de financiamiento de medicamentos es fundamental para lograr un sistema de salud equitativo. Se ha propuesto que los determinantes de la equidad en el financiamiento son socioeconómicos, demográficos y asociados a la intervención pública, sin embargo, se ha avanzado poco en su evaluación empírica y en la cuantificación de su importancia relativa. En este trabajo estimamos regresiones por cuantiles a nivel provincial en Argentina y encontramos que la población mayor a 65 años, el desempleo, la existencia de laboratorios públicos de producción de medicamentos, las transferencias de tratamientos y la orientación del sistema de salud a la atención primaria, son importantes predictores de la progresividad en el esquema de pagos. Ingresos bajos, instituciones débiles, infraestructura y provisión de servicios insuficientes redundan en respuestas sociales más regresivas a las necesidades sanitarias, empeorando las condiciones de vida y limitando las oportunidades de desarrollo.

Política Nacional de Medicamentos; Demografía; Factores

Socioeconómicos

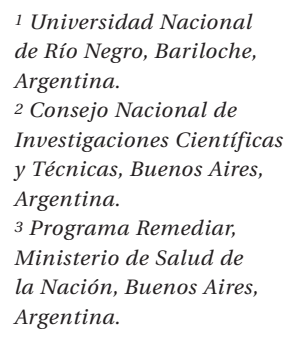

1 Universidad Nacional de Río Negro, Bariloche, Argentina.

2 Consejo Nacional de Investigaciones Científicas y Técnicas, Buenos Aires, Argentina.

3 Programa Remediar, Ministerio de Salud de la Nación, Buenos Aires, Argentina.

Correspondencia

M. Dondo

Universidad Nacional de Río Negro.

Mitre 630, 2c, Bariloche, Río Negro 8400, Argentina. marianadondo@gmail.com 


\section{Introducción}

La salud, como componente del capital humano, es un determinante del desarrollo de los países $1,2,3$. Por lo tanto, promover el acceso a la salud es una política del Estado orientada a mejorar, tanto la distribución de oportunidades, como la eficiencia productiva. Los medicamentos son bienes importantes dentro de la política de salud, ya que ayudan a restablecerla en casos de enfermedad y a mejorar la calidad de vida en casos de deterioro. En este sentido, ampliar el acceso a los medicamentos es un pilar importante dentro de las políticas de salud. Si bien se ha propuesto que el acceso suele estar limitado por barreras económicas, legales, culturales y geográficas 4 , se ha avanzado poco en la evaluación empírica de estas ideas, lo que resulta fundamental en el diseño de políticas de salud.

Una de las funciones de los sistemas de salud es garantizar la protección financiera, es decir, asegurar un financiamiento justo de la atención, evitar que las familias posterguen su atención por razones financieras y que incurran en gastos excesivos para atender sus necesidades de salud 5. Los medicamentos constituyen una parte importante del gasto de salud de los hogares 6,7, por lo tanto, tener un sistema de financiamiento de medicamentos equitativo es fundamental para lograr un sistema de salud equitativo. Alcanzar la equidad en salud significa que todas las personas tienen la oportunidad de alcanzar su potencial en salud, y que nadie debe estar en desventaja para hacerlo si eso es evitable 8,9. Con respecto al financiamiento, se considera equitativa una situación en la que las personas participan en el costo según su nivel de ingreso y no según sus riesgos en salud. Entonces, en un sistema equitativo, cada cual debería recibir recursos y oportunidades según sus necesidades y aportar según su capacidad de pago ${ }^{10}$. La distribución de costos de salud no debería empeorar la distribución del ingreso de una sociedad ${ }^{11} \mathrm{y}$ los gastos en salud no deberían ser empobrecedores 12,13. La salud suele financiarse de distintas fuentes: (a) públicamente mediante impuestos (directos e indirectos); (b) seguros de salud; y (c) pagos de bolsillo. La equidad del sistema de financiamiento depende de la progresividad de todas estas fuentes. En términos generales, se ha propuesto que los sistemas basados más fuertemente en pagos de bolsillo suelen ser más regresivos o inequitativos, mientras que los esquemas financiados por la seguridad social son progresivos si los más ricos están incluidos en este sistema de protección ${ }^{10}$.

Se ha planteado que los determinantes de la equidad en el financiamiento de bolsillo de los medicamentos suelen ser socioeconómicos, demográficos o relacionados con la intervención pública. Sin embargo, existen pocos trabajos empíricos que cuantifiquen el efecto de estos determinantes (entre otros, ver 5,6,7,14,15) y falta avanzar para comprender la importancia relativa de estos determinantes, información de gran relevancia para el diseño de políticas públicas. Como determinantes socioeconómicos suelen mencionarse el nivel y la distribución de los ingresos, el nivel de empleo, de pobreza, de cobertura formal de salud, el perfil epidemiológico de la población y los precios de los medicamentos 5,13,16. Se propone que deterioros en estas variables generen peores resultados en la equidad en el financiamiento, y además, como entre las variables hay asociaciones, el efecto de la desmejora podría potenciarse, asemejándose a la "trampa de la pobreza”. Ésta hace referencia a que los pobres se enferman más y esto impacta negativamente en sus ingresos laborales, el pago de los tratamientos se hace muchas veces a costa de otros pagos, como la educación de los hijos, perpetuando la pobreza inter-generacionalmente ${ }^{9,11}$.

En cuanto a la dimensión demográfica, se suelen mencionar la estructura de edades de la población, la densidad de la población y el nivel de urbanización como posibles determinantes de la equidad 5,14,16. La estructura demográfica puede afectar de formas contrastantes a la equidad en el financiamiento de los medicamentos. Por un lado, se plantea que poblaciones con mayor porcentaje en grupos de edad más vulnerables y demandantes de medicamentos, como los adultos mayores o los niños que, adicionalmente, están en etapas pasivas (no generan ingresos), podrían tener peores resultados de equidad en el financiamiento. Sin embargo, el impacto podría ser el opuesto, si el gasto público -destinado a la provisión de medicamentos- se focalizara según la necesidad, medida por la mayor presencia de estos grupos más vulnerables. En este caso, una mayor proporción de estos grupos en la estructura demográfica se relacionaría con una mayor equidad si el gasto fuera efectivo. Por otro lado, la estructura demográfica de la población y el nivel de desarrollo suelen estar relacionados, de modo que a mayor nivel de desarrollo suele asociarse una población más envejecida (más adultos mayores y menos niños). El mayor peso de los adultos mayores podría impactar en un sistema de financiamiento más regresivo 17, pero la menor presencia de niños se vincularía, por el contrario, a un sistema más progresivo. De este modo, si bien parece claro que la estructura demográfica afecta la progresividad en el financiamiento de los medicamentos, aún es necesario aportar evidencia empírica acerca de cuál es el impacto 
que prepondera, con el fin de diseñar sistemas de protección adecuados. En relación a la densidad y urbanización, población más dispersa suele tener menos acceso a los servicios de salud y a los medicamentos por las barreras geográficas ${ }^{4}$. Es más costoso implementar servicios públicos en las zonas rurales y los programas estatales suelen centrarse en ámbitos urbanos. Por lo tanto, el acceso a estos bienes y servicios suele ser menor en las zonas rurales. Estos aspectos demográficos (el nivel de urbanización y la densificación) también están vinculados al nivel de desarrollo económico.

En cuanto a los determinantes, relacionados con la intervención pública, se pueden mencionar la amplitud de la red de atención de oferta pública de bienes y servicios de salud, la orientación a la atención primaria de esta red, las transferencias en especie y la producción pública de medicamentos. Diferentes visiones acerca del rol del Estado proponen hipótesis alternativas acerca de la relación entre la intervención pública y la equidad en salud. Por un lado, planteamientos liberales proponen que se puede alcanzar una mayor equidad fortaleciendo los mercados, es decir, facilitando una mayor competencia en el financiamiento y la prestación de servicios de salud y el suministro de insumos para bajar sus costos y mejorar su calidad 18,19. Por otro lado, posturas más heterodoxas plantean que cuanto más amplia sea la red de atención pública de la salud, mejor será el acceso a los medicamentos gratuitos de la población más pobre, y por lo tanto, mayor será la equidad en el financiamiento $10,20,21$. Las transferencias en especie podrían disminuir el porcentaje de ingresos destinados al gasto en medicamentos de la población más pobre, generando así mayor progresividad en el sistema de financiamiento ${ }^{6}$. Para lograrlo, las transferencias deberían considerar algunas medidas complementarias: (a) un listado de medicamentos esenciales, para garantizar la selección de medicamentos por criterios de costo-efectividad; (b) protocolos de prescripción y tratamiento para tender al uso racional; (c) el establecimiento de controles para evitar desvíos y desperdicios en el sistema de distribución; (d) la compra en gran escala para obtener mejores precios; y (e) la focalización hacia los más pobres o sin cobertura. La existencia de laboratorios públicos, por su parte, implicaría mayor disponibilidad de medicamentos gratuitos para la población que se atiende en el subsector público, mejorando la equidad 7 . Adicionalmente, conocer el proceso de producción de medicamentos puede brindarle al Estado información necesaria para regular mejor este mercado.
Argentina se presenta como un caso interesante para el estudio de los determinantes de la equidad en el financiamiento de los medicamentos, dado que tiene una gran disparidad espacial, tanto en la necesidad o demanda potencial de medicamentos, relacionada con variables socioeconómicas y demográficas, como en la oferta de bienes y servicios de salud. El sistema de salud argentino se compone de 3 subsectores: el público, el de la seguridad social y el privado. El Ministerio de Salud es responsable y garante del derecho a la salud, sin embargo, como los gobiernos provinciales son autónomos, en la práctica deciden de manera independiente las políticas de salud a implementar en sus territorios de influencia. Las estrategias de salud pública de cada jurisdicción suelen atender a iniciativas individuales y a las necesidades de su población, dependiendo de su estructura de oferta, de la normativa jurisdiccional y del peso relativo de los distintos actores en el financiamiento y la provisión de servicios 22 . Ésto podría generar acentuadas disparidades entre provincias en el acceso a los servicios de salud y en la cobertura y calidad de los servicios prestados. Por otro lado, desde al año 2002 Argentina cuenta con la Política Nacional de Medicamentos, que comprende la Ley de Promoción de la Utilización de Medicamentos por Nombre Genérico (Ley n. 25.649/02) 23, el Programa Médico Obligatorio (selectividad en la financiación por los seguros de salud) y el Programa Remediar. La política incluye, entonces, elementos de regulación, generación y difusión de información y provisión gratuita de medicamentos esenciales. Estas medidas buscan, por un lado, fortalecer la competencia por precios en el mercado de medicamentos (en detrimento de la competencia por marcas), haciendo descender su precio hacia el costo medio de producción y disminuyendo la dispersión de precios existente entre medicamentos equivalentes de diferente marca comercial. Por otro lado, están orientadas a estimular el copago de las obras sociales 24 . El valor del copago es un porcentaje del gasto total que paga el usuario (o en algunos casos, un monto fijo), complementado el pago realizado por otro pagador, que también es financiado por el usuario en forma de seguro sea social o privado. Adicionalmente, las medidas implementadas amplían la posibilidad de elección del paciente, habilitando la sustitución de medicamentos por marcas más baratas 25. Como consecuencia de todas estas intervenciones, el gasto de bolsillo en medicamentos de los hogares más pobres debería disminuir, ampliando el acceso y favoreciendo la equidad. Si bien la política es de aplicación nacional, los patrones provinciales pueden influir en sus efectos sobre la equidad. 
Adicionalmente, en Argentina existen pocos estudios acerca de la equidad en el financiamiento de los medicamentos 6,18,26,27 y faltan estudios empíricos que convaliden las ideas acerca de cuáles son sus determinantes. Tampoco se hicieron estudios que analicen las dimensiones demográfica, socioeconómica y relacionada a la intervención pública simultáneamente, ni que permitan conocer el aporte relativo de cada una de estas a la equidad en el financiamiento privado de los medicamentos (es decir, pagos de bolsillo). En este trabajo buscamos avanzar en ese sentido, estudiando cuáles son los factores que influyen a nivel provincial en la equidad en el financiamiento de medicamentos por parte de los hogares en Argentina. Los resultados que presentamos permiten evaluar la importancia relativa de cada uno de estos factores para promover sistemas de financiamiento y, por lo tanto, sistemas de salud, más equitativos.

\section{Métodos}

Variables, indicadores y fuentes de información

Consideramos más equitativo un sistema de financiamiento del gasto de bolsillo en medicamentos más progresivo y medimos la progresividad a través del índice de Kakwani (IK), que compara el aporte de un grupo al gasto en medicamentos, en relación a la porción de ingreso nacional que percibe ${ }^{28}$. El IK se mide como el área entre dos curvas, que reflejan respectivamente: (1) la proporción acumulada de ingresos percibido por cada grupo de población (ordenada por nivel de ingresos); (2) la concentración del pago aportado por cada grupo de población (ordenada por nivel de ingresos). Si los pagos por medicamentos se recaudan en proporción al ingreso, las dos curvas coinciden y el IK toma el valor cero. Si el sistema es progresivo, es decir, que la contribución al pago aumenta con más rapidez que el ingreso, entonces la curva de concentración de pago queda por debajo de la curva de participación en el ingreso, el IK toma valores entre cero y uno. Si el sistema de financiamiento es regresivo (la tasa de pago disminuye con el ingreso), entonces la curva de pagos se ubica por encima de la curva de ingresos. Esto significa que grupos más pobres aportan mayor proporción de gasto de la que perciben del ingreso. El valor del IK en este caso toma valores entre cero y menos dos 29 .

La unidad de análisis fue la provincia y la fuente de información para calcular el IK la Encuesta de Gastos de los Hogares (ENGHo 20042005, Instituto Nacional de Estadística y Censos
- INDEC). Ésta releva la estructura de presupuesto de los hogares, el origen de los ingresos y su asignación a distintos gastos. Las unidades de recolección son las viviendas y los hogares, definidos como "una persona o grupo de personas que comparten una misma vivienda, bajo un régimen de tipo familiar, y consumen alimentos a cargo del mismo presupuesto". Los gastos se registran utilizando el criterio de adquirido (toma de posesión o adquisición del derecho a recibir) y el período de referencia varía según la cotidianeidad del consumo de bienes o servicios (por ej. períodos más cortos para alimentos y más largos para automóviles). El ingreso informado es el ingreso promedio percibido en los últimos 6 meses, y el ingreso del hogar suma los ingresos percibidos por todos los miembros. La muestra de la ENGHo 2004-2005 incluyó población residente en zonas urbanas y rurales.

En la Tabla 1 presentamos los indicadores utilizados para predecir la progresividad en el financiamiento de los medicamentos. Además, relevamos datos de localización de cada provincia (latitud y longitud del centroide de la provincia) del Instituto Geográfico Nacional. Las variables predictoras fueron utilizadas tanto en sus unidades de medida original, como estandarizadas a escala z. La transformación z es útil para comparar los efectos relativos de variables predictoras medidas en escalas diferentes y con diferentes metodologías y, adicionalmente, no modifica la forma de la relación funcional entre las variables predictoras y dependiente. La variable estandarizada expresa el número de desviaciones típicas que dista cada observación de la media.

\section{Análisis estadísticos}

En primer lugar, estimamos los coeficientes de correlación de Spearman para evaluar las asociaciones entre todas las variables. El coeficiente de correlación de Spearman es una medida de asociación lineal entre dos variables y toma valores entre $-1 \mathrm{y}+1$, donde el 0 indica que no existe variación conjunta entre las variables. Este método se recomienda cuando las variables estudiadas son ordinales o no cumplen con el supuesto de normalidad. Este análisis nos permitió seleccionar variables no multicolineales para incluir en el modelo de regresión (ver sección de Resultados).

Estimamos modelos de regresión por cuantiles (QR) para la mediana del IK, en función de las variables predictoras. Dado que QR no asume ninguna distribución residual (Koenker R. Quantreg: quantile regression. R package version 5.05. http: / /CRAN.R-project.org / package=quantreg), seleccionamos este método porque los datos de IK tenían varianzas heterogéneas y no estaban 
Indicadores utilizados para predecir la progresividad del sistema de financiamiento de los medicamentos a nivel provincial, estimada mediante el índice de Kakwani (IK).

\begin{tabular}{|c|c|c|c|}
\hline Indicador & Justificación & $\begin{array}{c}\text { Asociación } \\
\text { esperada con el IK }\end{array}$ & $\begin{array}{l}\text { Fuente de } \\
\text { información }\end{array}$ \\
\hline \multicolumn{4}{|l|}{ Dimensión socioeconómica } \\
\hline $\begin{array}{l}\text { Cobertura: \% de habitantes con algún } \\
\text { tipo de cobertura de salud (prepaga u } \\
\text { obra social) }\end{array}$ & $\begin{array}{l}\text { La población con cobertura de salud cuenta con un tercer } \\
\text { pagador que co-financia los medicamentos y por eso deben } \\
\text { financiar menos de su bolsillo. Es decir, la cobertura disminuye } \\
\text { el precio que los consumidores pagan en el mercado y, por } \\
\text { eso, aumenta la posibilidad de acceso. }\end{array}$ & Positiva & $\begin{array}{l}\text { INDEC- Censo } \\
\text { Nacional, } 200148\end{array}$ \\
\hline $\begin{array}{l}\text { Desempleo: \% de habitantes } \\
\text { desocupados }\end{array}$ & $\begin{array}{l}\text { Mayor desempleo implica menores ingresos y menor nivel de } \\
\text { cobertura de salud. }\end{array}$ & Negativa & \\
\hline $\begin{array}{l}\text { Pobreza: \% de hogares pobres por } \\
\text { necesidades básicas insatisfechas }\end{array}$ & $\begin{array}{l}\text { Los hogares pobres tienen menores ingresos (y por eso menor } \\
\text { capacidad de aseguramiento formal), peores resultados en } \\
\text { salud y enfermedades más prolongadas. El peso del gasto de } \\
\text { bolsillo en medicamentos, en relación a sus ingresos, es mayor. }\end{array}$ & Negativa & \\
\hline $\begin{array}{l}\text { Perfil_E: toma los valores del } 1 \\
\text { (mejor perfil epidemiológico) al 4, en } \\
\text { función de los AVPP por infecciones } \\
\text { o tumores * }\end{array}$ & $\begin{array}{l}\text { Un mayor valor de perfil epidemiológico implica mayor } \\
\text { demanda de medicamentos y, adicionalmente, está } \\
\text { relacionado con mayor pobreza y menor posibilidad de } \\
\text { aseguramiento formal. }\end{array}$ & Negativa & Maceira 12 \\
\hline \multicolumn{4}{|l|}{ Dimensíon demográfica } \\
\hline $\begin{array}{l}\text { Mayores: \% de habitantes mayores de } \\
65 \text { años de edad }\end{array}$ & Este grupo de edad es más demandante de medicamentos. & Negativa & $\begin{array}{l}\text { INDEC- Censo } \\
\text { Nacional, } 200148\end{array}$ \\
\hline $\begin{array}{l}\text { Menores: \% de habitantes menores } \\
\text { de } 14 \text { años de edad }\end{array}$ & $\begin{array}{l}\text { Este grupo de edad es más demandante de medicamentos } \\
\text { y adicionalmente en los hogares más pobres suele haber } \\
\text { más menores de edad (ver justificación de Pobreza). }\end{array}$ & Negativa & \\
\hline Dens_Pob: número de habitantes $\mathrm{km}^{-2}$ & $\begin{array}{l}\text { Población más dispersa suele tener menor acceso al sistema } \\
\text { por barreras geográficas. }\end{array}$ & Positiva & \\
\hline Pob_urbana (\%) & $\begin{array}{l}\text { Servicios públicos son más costosos de implementar en las } \\
\text { zonas rurales y los programas estatales se suelen centrar en } \\
\text { ámbitos urbanos. Por lo tanto, el acceso a estos bienes y } \\
\text { servicios suele ser menor en las zonas rurales. }\end{array}$ & Positiva & \\
\hline
\end{tabular}

(continua)

normalmente distribuidos. Los coeficientes de regresión parciales para las variables en su escala original y en escala $\mathrm{z}$ fueron estimados mediante la función rq del paquete Quantreg del programa R (Koenker R. Quantreg: quantile regression. R package version 5.05. http://CRAN.R-project.org/ package $=$ quantreg). Estimamos los modelos que surgen de todas las combinaciones posibles de las variables predictoras y los ordenamos en función del criterio de información de Akaike (AIC) 29,30, utilizando la función dredge del paquete MuMIn del programa R (Bartons K. Mukti-model Inference. R package version 1.12.1. http:// CRAN.R-project.org/package=MuMIn). El AIC es un criterio ampliamente utilizado para comparar hipótesis (modelos) competitivas. El valor del AIC se calcula en relación a dos componen- tes del modelo: (1) el ajuste relativo a los datos (mediante valores de máxima verosimilitud) y (2) su complejidad (número de parámetros) 31. Cuanto más bajo el AIC, se considera más parsimonioso el modelo. También calculamos la variación del AIC $(\triangle \mathrm{AIC})$, que mide la diferencia entre el AIC de cada modelo y el mínimo AIC encontrado (modelo más parsimonioso). Valores de $\triangle$ AIC por encima de 7 indican que el modelo tiene una bondad de ajuste pobre en relación al mejor modelo, mientras que valores por debajo de 2 indican que los modelos son indistinguibles entre sí 32 . Adicionalmente, usamos los valores de AIC para obtener los pesos de Akaike $\left(\mathrm{w}_{\mathrm{i}}\right)$ de cada modelo, lo que nos permitió calcular la importancia relativa de cada variable predictora. Lo realizamos sumando los $\mathrm{w}_{\mathrm{i}}$ de todos los modelos 
Tabla 1 (continuación)

\begin{tabular}{|c|c|c|c|}
\hline Indicador & Justificación & $\begin{array}{c}\text { Asociación } \\
\text { esperada con el IK }\end{array}$ & $\begin{array}{l}\text { Fuente de } \\
\text { información }\end{array}$ \\
\hline \multicolumn{4}{|l|}{$\begin{array}{l}\text { Dimensión de oferta de bienes y } \\
\text { servicios de salud }\end{array}$} \\
\hline $\begin{array}{l}\text { Laboratorio: toma el valor } 1 \text { en el } \\
\text { caso de que haya un laboratorio } \\
\text { público en la provincia. }\end{array}$ & $\begin{array}{l}\text { La existencia de un laboratorio público implicaría una mayor } \\
\text { disponibilidad de medicamentos gratuitos para población que } \\
\qquad \text { se atiende en el subsector público **. }\end{array}$ & Positiva & Apella 7 \\
\hline $\begin{array}{l}\text { Transferencias: suma anual de las } \\
\text { transferencias realizadas por Remediar } \\
\text { a los centros de salud, en términos } \\
\text { de costo de adquisición (millones de } \\
\text { USD) y de millones de tratamientos }\end{array}$ & $\begin{array}{l}\text { Un aumento de las transferencias reduciría el porcentaje de } \\
\text { ingreso destinado al gasto en medicamentos del grupo de } \\
\text { población más pobre }{ }^{\star \star \star} \text {. }\end{array}$ & Positiva & $\begin{array}{l}\text { Base de datos } \\
\text { de Remediar, } \\
\text { Ministério de la } \\
\text { Salud } 24\end{array}$ \\
\hline $\begin{array}{l}\text { Hospitales: número de hospitales } \\
\text { cada } 100 \text { mil habitantes }\end{array}$ & $\begin{array}{l}\text { Una red de atención pública de la salud más amplia mejora el } \\
\text { acceso a los medicamentos de la población más pobre. }\end{array}$ & Positiva & Tobar et al. 49 \\
\hline CAPS: por cada 100 mil habitantes & & Positiva & \\
\hline
\end{tabular}

AVPP: años de vida potencialmente perdidos; CAPS: Centro de Atención Primaria de la Salud.

* Los AVPP se midieron cada 10 mil habitantes en 1999. La categoría 1 agrupa provincias con AVPP por tumores e infecciones por debajo de la media nacional; la categoría 2 agrupa provincias con AVPP por tumores por encima y AVPP por infecciones por debajo de la media nacional; la categoría 3 se compone de provincias con AVPP por infecciones por encima pero AVPP por tumores por debajo de la media nacional; y la categoría 4 se compone de aquellas provincias cuyos AVPP por ambas causas se encuentran encima de la media nacional;

** Los laboratorios provinciales son estatales de jurisdicción nacional, provincial o municipal o de universidades públicas. Estos laboratorios no venden sus productos en el mercado, sino que los utilizan para la dispensa en sus sistemas de salud, para satisfacer principalmente la demanda de población pobre y sin cobertura de salud en sus territorios de influencia. Se ha comprobado (Apella 7) que los precios de mercado de los medicamentos son superiores a los costos de los laboratorios públicos (en el año 2003, el costo unitario promedio de los medicamentos producidos en laboratorios públicos era un 63.5\% menor a su precio de mercado);

*** Las transferencias de medicamentos del programa Remediar tienen en cuenta el perfil epidemiológico y además buscan reponer el uso que se dio a los medicamentos en cada Centro de Atención Primaria de la Salud (CAPS), por lo tanto, es una aproximación a la necesidad de uso de cada población.

El montaje y distribución de botiquines de medicamentos se realiza en función de un sistema de información que permite asignar botiquines diferenciados que contemplan la existencia de distintos segmentos de CAPS (según recetas mensuales producidas, por ejemplo), la heterogeneidad en la utilización de determinados medicamentos (heterogeneidad estacional, regional), entre otros.

en los que estaba incluida la variable predictora de interés, utilizando la función "importance" del paquete MuMIn del programa R (Bartons K. Mukti-model Inference. R package version 1.12.1. http://CRAN.R-project.org/package=MuMIn). Verificamos la ausencia de multicolinealidad entre las variables predictoras de los modelos de mejor ajuste, estimando el factor de inflación de la varianza (FIV) (función vif del paquete car del programa $\mathrm{R}$ 33). Valores de FIV mayores a 10 indican la existencia de multicolinealidad entre las variables, lo que podría introducir sesgos en las estimaciones 34 . Finalmente, evaluamos la autocorrelación espacial del modelo con menor AIC, mediante el Índice de Moran sobre los residuos, para evaluar el supuesto de independencia (función MoranI del paquete ape del programa R 35). Utilizamos una matriz de pesos en la cual los elementos de la diagonal son ceros y los elementos fuera de la diagonal son: 1 / (distancia entre el punto i y el j) ${ }^{35}$. Los valores de pares de puntos que se ubican más cerca son mayores a los pun- tos ubicados más distantes entre sí. Si hubiera una asociación espacial (por ej., provincias más cercanas son más similares entre sí que las más apartadas), entonces la localización podría ser un factor influyente en estas relaciones y, en caso de no considerarlo, los parámetros estimados estarían sesgados 36 .

\section{Resultados}

Con excepción de Misiones, en todas las provincias el sistema de financiamiento de medicamentos fue regresivo, es decir, el IK fue negativo (Figura 1). La provincia de Chaco fue la que tuvo el sistema más regresivo (IK = -0,255). En Misiones, el índice tomó un valor muy cercano a cero $(\mathrm{IK}=0,006)$ indicando que el esquema fue proporcional (la concentración de gastos es muy similar a la concentración de ingresos). Encontramos una gran disparidad espacial, tanto en las variables socioeconómicas y demográficas, 
Figura 1

Progresividad del sistema de financiamiento de los medicamentos a nivel provincial en 2005, estimada mediante el índice de Kakwani.

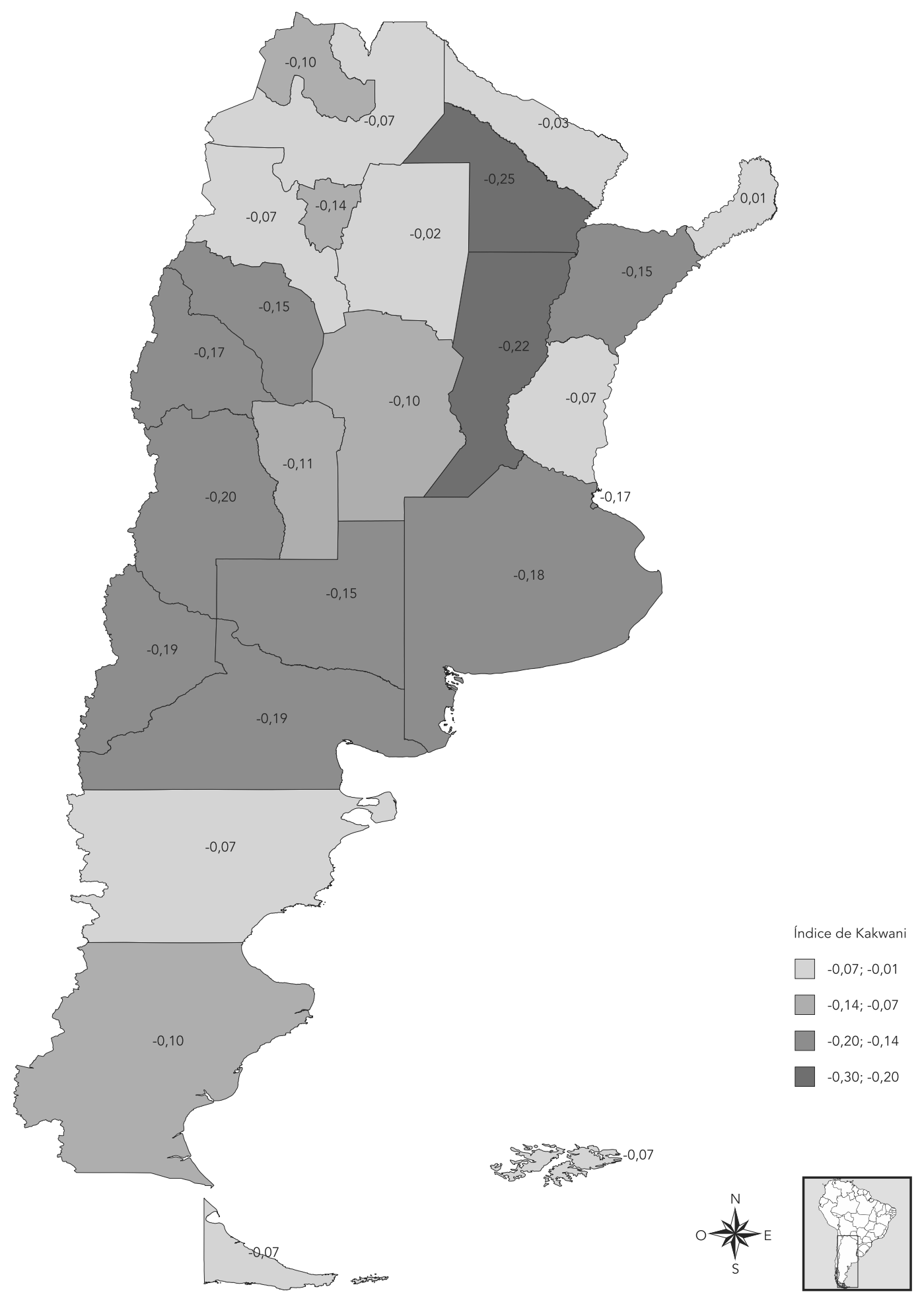

CAPS: Centro de Atención Primaria de la Salud. 
como en la oferta de bienes y servicios de salud a nivel provincial (Tabla 2).

Considerando los coeficientes de correlación de Spearman $(r)$ entre las variables predictoras, excluimos de la estimación de los modelos de regresión las siguientes variables: (a) Menor: fuerte y negativamente correlacionada con Mayor $(r=$ $-0,81)$ y Cobertura $(r=-0,62)$ y, positivamente, asociada a Pobreza $(r=0,87)$; (b) Pobreza: fuerte y negativamente asociada a Cobertura $(r=-0,69)$ y Pob_urbana $(r=-0,65)$ y positivamente asociada a Menor $(r=0,87)$ y Perfil_E $(r=0,64)$; y (c) Dens_ Pob: positivamente asociada a Transferencias $(r=0,84)$ y negativamente asociada a Hospital $(r=-0,69)$. Excluidas estas variables predictoras, los modelos de menor AIC obtenidos en la QR se presentan en la Tabla 3. Los coeficientes y errores estándar corresponden a las variables estandarizadas, lo que permite comparar sus magnitudes. Los modelos con menor AIC resultantes de la estimación con las variables en escalas originales incluyeron las mismas variables predictoras, y los signos de los coeficientes de regresión parcial coincidieron con los de variables estandarizadas (resultados no presentados). Los valores de los FIVs indican que no hay multicolinealidad entre las variables predictoras, y tampoco encontramos evidencia de autocorrelación espacial en los residuos del modelo con menor AIC (Moran's $I=$ $-0,08$; valor de $\mathrm{p}=0,64$ ).

Los indicadores Mayor, Desempleo, Laboratorio, Transferencias y CAPS son predictores de la equidad en el financiamiento de los medicamentos (Tabla 3), y los signos de sus coeficientes de regresión coincidieron con los esperados (Tabla 1). Entre estos, los indicadores con mayor importancia predictiva sobre la progresividad en el financiamiento de medicamentos fueron Desempleo, Mayores y Laboratorio (Figura 2). El indicador socioeconómico Desempleo se asoció negativamente al IK, mostrando que cuando el desempleo aumenta en una unidad de desviaciones estándar (manteniendo el resto de las variables constantes), el IK empeora en 1,07 unidades

Tabla 2

Mediana, mínimo y máximo observados para cada uno de los indicadores utilizados para predecir la progresividad del sistema de financiamiento de los medicamentos a nivel provincial en la Argentina.

\begin{tabular}{|c|c|c|c|}
\hline Indicador & Mediana & Mínimo & Máximo \\
\hline \multicolumn{4}{|l|}{ Dimensión socioeconómica } \\
\hline Cobertura (\%) & 51,2 & 34,2 (Formosa) & 73,8 (CABA) \\
\hline Desempleo (\%) & 14,0 & 9,8 (Santa Cruz) & 19,2 (Buenos Aires) \\
\hline Pobreza (\%) & 15,5 & 7,1 (CABA) & 28,0 (Formosa) \\
\hline Perfil_E & \multicolumn{3}{|c|}{$\begin{array}{l}6 \text { provincias se encuentran en la categoría 1; } 6 \text { en la categoría 2; } \\
11 \text { en la categoría } 3 \text { y sólo } 1 \text { (Chaco) en la categoría } 4\end{array}$} \\
\hline \multicolumn{4}{|l|}{ Dimensión demográfica } \\
\hline Mayores (\%) & \multicolumn{2}{|r|}{ Fuego) } & 17,2 (CABA) \\
\hline Menores (\%) & 32,0 & $16,9(\mathrm{CABA})$ & 37,0 (Misiones) \\
\hline Dens_Pob (hab. km-2) & 6,9 & 0,8 (Santa Cruz) & 13680 (CABA) \\
\hline Pob_urbana (\%) & 83,9 & Estero) & 100 (CABA) \\
\hline \multicolumn{4}{|l|}{$\begin{array}{l}\text { Dimensión de oferta de bienes y } \\
\text { servicios de salud }\end{array}$} \\
\hline Laboratorio & \multicolumn{3}{|c|}{$\begin{array}{c}14 \text { provincias argentinas tenían laboratorio público en el año 2005: } \\
\text { CABA, Buenos Aires, Córdoba, Entre Ríos, Formosa, La Pampa, } \\
\text { Mendoza, Misiones, Río Negro, Salta, San Juan, San Luis, } \\
\text { Santa Fe y Tucumán. }\end{array}$} \\
\hline Transferencia (millones de USD año-1) * & 17,1 & 9,7 (Santa Cruz) & 37,2 (La Pampa) \\
\hline Hospitales (número 100 mil hab-1) & 5,4 & $1,0(\mathrm{CABA})$ & 11,7 (Catamarca) \\
\hline CAPS (número 100 mil hab-1) & 18,8 & 1,8 (CABA) & $\begin{array}{l}\text { 35,5 (Santiago del } \\
\text { Estero) }\end{array}$ \\
\hline
\end{tabular}

CABA: Ciudad Autónoma de Buenos Aires.

* En este caso se presenta el dato de Transferencia en tratamientos por habitante con necesidades básicas insatisfechas. 


\section{Tabla 3}

Coeficientes estimados para la regresión por cuantiles en la predicción del índice de Kakwani (IK) a nivel provincial (errores estándar entre paréntesis) y criterio de información de Akaike (AIC). El $\triangle \mathrm{AIC}$ muestra la diferencia entre el AIC de un determinado modelo y el de mejor ajuste. Las variables predictoras fueron estandarizadas usando la escala z para permitir la comparación de los valores de los coeficientes de regresión parcial.

\begin{tabular}{|c|c|c|c|c|c|c|c|}
\hline Indicador\Modelo & Mejor ajuste & A & B & C & D & $E$ & Nulo \\
\hline \multicolumn{8}{|c|}{ Dimensión socioeconómica } \\
\hline Cobertura & & & & $-0,12(0,2)$ & & & \\
\hline Desempleo & $-1,07(0,31)$ & $-0,83(0,33)$ & $-1,01(0,25)$ & $-0,97(0,32)$ & $-1,07(0,34)$ & $-0,84(0,44)$ & \\
\hline Perfil E & & $0,29(0,13)$ & $0,17(0,16)$ & & & & \\
\hline \multicolumn{8}{|c|}{ Dimensión demográfica } \\
\hline Mayores & $-0,74(0,13)$ & $-0,46(0,22)$ & $-0,65(0,12)$ & $-0,62(0,19)$ & $-0,67(0,16)$ & $-1,00(0,35)$ & \\
\hline Pob_urbana & & & & & $-0,04(0,24)$ & & \\
\hline \multicolumn{8}{|c|}{$\begin{array}{l}\text { Dimensión de oferta de } \\
\text { bienes y servicios de salud }\end{array}$} \\
\hline Laboratorio & $1,62(0,47)$ & $1,28(0,53)$ & $1,50(0,24)$ & $1,62(0,58)$ & $1,54(0,58)$ & $1,57(0,66)$ & \\
\hline Transferencias & $0,44(0,17)$ & $0,30(0,16)$ & $0,32(0,15)$ & $0,31(0,17)$ & $0,42(0,19)$ & $0,49(1,85)$ & \\
\hline Hospitales & $-0,44(0,33)$ & & $-0,30(0,30)$ & $-0,51(0,37)$ & $-0,46(0,33)$ & & \\
\hline CAPS & $0,73(0,30)$ & $0,48(0,26)$ & $0,42(0,22)$ & $0,64(0,34)$ & $0,68(0,33)$ & $0,77(0,31)$ & \\
\hline $\mathrm{AIC}$ & 63 & 64,1 & 64,4 & 64,4 & 64,8 & 65,2 & 75,2 \\
\hline$\Delta \mathrm{AIC}$ & 0,0 & 1,1 & 1,4 & 1,4 & 1,8 & 2,2 & 12,2 \\
\hline FIV & $<3,2$ & $<2,1$ & $<3,3$ & $<3,3$ & $<3,5$ & $<2,1$ & \\
\hline
\end{tabular}

CAPS: Centro de Atención Primaria de la Salud; FIV: factor de inflación de la varianza.

Figura 2

Importancia relativa de cada indicador en la predicción del índice de Kakwani (IK) a nivel provincial. La altura de cada barra es la suma de los pesos de Akaike de todos los modelos que incluyeron esa variable predictora. Los colores de las barras varían según representen las dimensiones socioeconómica, demográfica o de intervención pública.

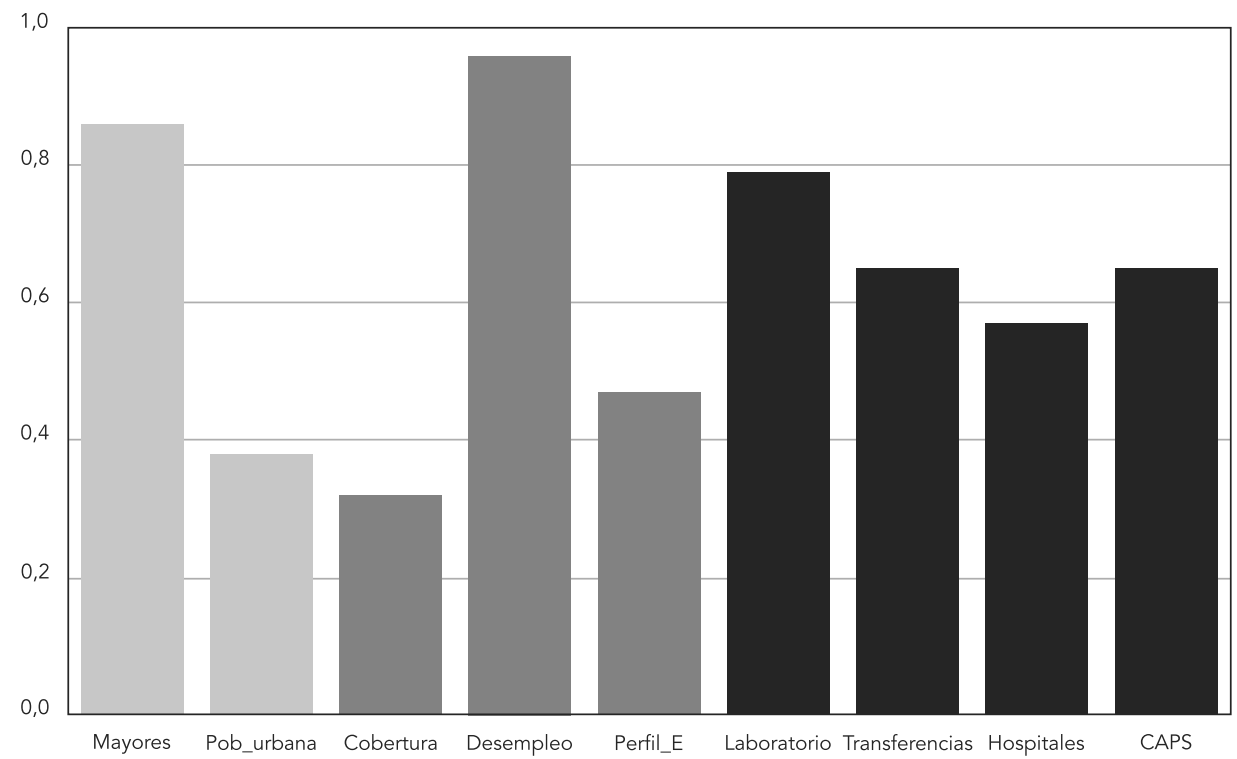

CAPS: Centro de Atención Primaria de la Salud. 
de desviación estándar (Tabla 3). El coeficiente que acompaña al indicador demográfico Mayores mostró que a mayor población adulta en una provincia, es menor la equidad en el financiamiento de los medicamentos (Tabla 3). Adicionalmente, como la población de mayores se asoció fuerte y negativamente con la población de menores, se puede plantear que en situaciones con más mayores y menos menores el sistema de financiamiento será más regresivo. A priori esperabamos el resultado opuesto para el porcentaje de menores (Tabla 1), ya que en general hay más menores en los hogares más pobres (el $r$ entre Pobreza y Menores fue de 0,87 ). Con respecto a los indicadores relacionados con la intervención del Estado (Laboratorio, Transferencias y CAPS), los mismos señalaron que cuanto mayor es la oferta de bienes y servicios de salud pública en un territorio, mejor es la progresividad del sistema de financiamiento de medicamentos (Tabla 3). Las otras variables regresoras (Pob_urbana, Cobertura, Perfil_E, Hospitales) quedaron incluidas sólo en algunos de los modelos de menor AIC y, además, tuvieron coeficientes de regresión parcial cuyos intervalos de confianza al $90 \%$ incluian al cero. Por lo tanto, la evidencia que encontramos respecto de su importancia como predictoras de la equidad medida por el IK no es fuerte (Tabla 3 y Figura 2).

\section{Discusión y conclusiones}

El acceso a los medicamentos depende del ingreso, los precios y del modelo de organización del sistema de salud, que delimita el rol del sector público en esta área y las barreras económicas que suponen los esquemas de copagos. En este trabajo avanzamos en la evaluación empírica de las asociaciones entre las dimensiones socioeconómica, demográfica y de intervención pública con la equidad en el financiamiento de los medicamentos en Argentina a nivel provincial. Encontramos grandes diaparidades, tanto en los determinantes, como en la equidad en el financiamiento de bolsillo de medicamentos a nivel subnacional. La teoría del federalismo fiscal propone que descentralizar las políticas de salud tendría ventajas como las de conocer mejor las demandas del ciudadano, permitiendo adaptar las intervenciones a las preferencias y necesidades, lograr una mayor participación y rendición de cuentas y profundizar los procesos de democratización ${ }^{37}$. Sin embargo, las asimetrías provinciales encontradas resaltan la importancia de complementar las políticas específicas del ámbito de la salud con instrumentos que contribuyan a disminuir las desigualdades subnacionales 37 , de modo que igualen oportunidades entre los residentes. Ejemplos de estos instrumentos que se están implementando en Argentina son las transferencias de medicamentos (Remediar) y la reglamentación de la Ley de Producción Pública (Ley $n$. 26.688) 38, orientada a lograr una coordinación de los laboratorios provinciales para la producción de medicamentos esenciales y fomentar su tránsito federal, con el fin de aprovechar las economías de escala y ofrecerlos a un costo aún menor y respondiendo a las necesidades epidemiológicas.

Con respecto a los indicadores socioeconómicos, encontramos que tener más desempleo resultó en un esquema de gasto de bolsillo más regresivo y éste fue el indicador de mayor importancia relativa en la predicción de la equidad (Figura 2). Mayores niveles de desempleo estarían asociados a una mayor inequidad en el financiamiento de los medicamentos por múltiples vías. Primero, disminuyendo el nivel de ingreso disponible en los hogares para gastos corrientes en medicamentos. Segundo, reduciendo el nivel de cobertura formal de salud, que funciona como tercer pagador que cofinancia los medicamentos y, por lo tanto, baja el precio que los consumidores pagan en el mercado. Finalmente, si el desempleo se prolonga, suele generar mayor pobreza que, a su vez, se asociaría a peores perfiles epidemiológicos (peores resultados de salud y enfermedades más prolongadas) y hogares con más cantidad de miembros (en particular, niños). De este modo, los desempleados deben afrontar mayores gastos relativos a su ingreso en medicamentos para restaurar la salud sin seguros que cofinancien, con enfermedades más prolongadas y con menores ingresos disponibles 4,9,11.

Encontramos que los factores demográficos influyen fuertemente en la equidad del esquema de pagos y, por lo tanto, deben ser considerados en el diseño de los sistemas de protección social. En particular, estructuras demográficas con mayor porcentaje de mayores a 65 años tendrán esquemas de financiamiento relativamente más regresivos, ya que tienen mayor proporción de población que es pasiva en el mercado de trabajo y a la vez muy demandante de medicamentos por tener una mayor prevalencia de enfermedades 15,18. A su vez, más población de mayores se asoció con menos población de menores a 14 años de edad, vinculada fuertemente a una menor pobreza y mayor cobertura de salud, indicadores que suelen ser utilizados para focalizar el gasto público. Por lo tanto, la inequidad asociada a una menor porción de la población de niños podría explicarse por el gasto público en salud, asignado para atender a esta población. El dise- 
ño de sistemas de protección que garanticen el acceso a la salud y los medicamentos necesarios a los adultos mayores implica un gran desafío en sociedades latinoamericanas que presentan dinámicas demográficas tendientes al envejecimiento de su población 39 .

Existe un amplio debate en torno al rol que corresponde al Estado en la economía. Algunos autores plantean que su intervención debería ser mínima para lograr una mayor eficiencia y equidad, interviniendo únicamente para corregir las fallas de mercado 19,20. En particular, proponen que la salud corresponde al ámbito privado, que la información que existe es completa y está perfectamente distribuida y asumen que los consumidores, que tienen diferentes necesidades y preferencias y hacen elecciones racionales, obtendrán la mayor satisfacción si pueden elegir la canasta de bienes que quieren adquirir, pagando por lo percibido en el mercado. Por eso plantean que el mercado por si solo genera el mayor bienestar social y la asignación de recursos más eficiente. Sin embargo, en el sistema de salud se encuentran muchos bienes públicos o meritorios, los mercados tienen muchos fallos y además no se preocupan por alcanzar resultados equitativos $20,40,41$. La incidencia de eventos de enfermedad es incierta, los costos pueden ser catastróficos, y no existe un mercado de capitales que ofrezca préstamos por enfermedad, ya que no existen garantías de devolución. Considerando estas características, posturas más heterodoxas plantean que el Estado debe tener un rol activo en el mercado de medicamentos, interviniendo fuertemente para alcanzar el óptimo social 20,21. Estudios previos mostraron que la desregulación en el mercado de medicamentos no necesariamente se traduce en mayor competencia, menores precios y más eficiencia $42,43,44$ y que la falta de provisión pública limita el acceso a los más pobres, a los que residen en áreas menos urbanizadas o de baja densidad de población 4,20. En el año 2002 se implementó en Argentina la Ley de Promoción de la Utilización de Medicamentos por Nombre Genérico 23 que incluyó acciones de regulación sobre la información, los precios y la publicidad y buscó modificar las prácticas de prescripción. Se ha demostrado que la implementación de esta Ley contribuyó al fortalecimiento de la competencia en el mercado de medicamentos por precios en lugar de por marcas 45 , logrando descensos en los precios hacia sus costos medios de producción y también disminuciones en la dispersión de precios existente entre medicamentos equivalentes de diferente marca comercial 24,35. $\mathrm{Al}$ ampliar la posibilidad de elección del paciente, habilitando la sustitución de medicamentos por marcas más baratas, se lograron disminuir los gastos de bolsillo, aumentando el acceso de la población a los medicamentos y, por tanto, la equidad en su financiamiento. Nuestros resultados complementan estos estudios con un análisis espacial, apoyando esta última hipótesis, ya que las diferentes manifestaciones de intervención pública mostraron tener un efecto positivo sobre la equidad en el gasto de bolsillo en medicamentos. La estructura pública provincial de la oferta de salud, tanto en lo referente a la amplitud de la red de atención, como a su orientación a la atención primaria, tuvo un rol importante en la progresividad del gasto privado en medicamentos. Del mismo modo impactó la provisión pública nacional a través del programa Remediar y la provincial a través de la producción de laboratorios estatales, que contribuyeron a la disminución del porcentaje de ingresos destinados al gasto en medicamentos del grupo de población más pobre y sin cobertura.

En este trabajo aportamos evidencia empírica acerca de la asociación entre factores socioeconómicos, demográficos y de intervención pública, con la equidad en el financiamiento de los medicamentos, y cuantificamos la importancia relativa de los indicadores para predecirla. Nuestros resultados proponen que para ampliar el acceso a los medicamentos de la población que los necesite se requiere no sólo favorecer la intervención del Estado en el área de salud, sino también implementar estrategias que superan el ámbito específico de la salud, como las orientadas a mejorar las condiciones socioeconómicas de la población. El esquema de financiamiento debería lograr superar las inequidades resultantes del modelo de acumulación, ampliando el acceso también a los más pobres y los que tienen mayor riesgo en salud. Para ello se deberá buscar disminuir los pagos de bolsillo de medicamentos, a la vez que podría combinar el sistema contributivo solidario con sistemas no contributivos, basados en rentas generales que sean recaudadas de manera progresiva. El mayor acceso y cobertura en bienes y servicios de salud contribuye a mejorar el estado de salud de la población y, consecuentemente, impacta de manera positiva sobre la productividad y el desarrollo económico $1,2,3$. Por el contrario, las inequidades limitan las oportunidades de las personas de desarrollar su pleno potencial 8 y llevar la vida que desean. Ingresos bajos, instituciones débiles, infraestructura y provisión de servicios insuficientes, parecen redundar en respuestas sociales más regresivas a las necesidades sanitarias y, por tanto, empeoran las condiciones de vida y limitan las oportunidades del desarrollo 46,47 . 


\section{Colaboradores}

M. Dondo participó en el análisis e interpretación de los datos, la revisión bibliográfica y la redacción del artículo. M. Monsalvo contribuyó a la revisión crítica del artículo. L. A. Garibaldi participó en el análisis e interpretación de los datos y contribuyó a la revisión crítica del artículo.

\section{Referencias}

1. Barghava A, Jamison DT, Lau LJ, Murray CJL. Modeling the effects of health on economic growth. J Health Econ 2001; 20:423-40.

2. Bloom D, Canning D. The health and wealth of nations. Science 2000; 287:1207-9.

3. Weil D. Accounting for the effect of health on economic growth. Q J Econ 2007; 122:1265-306.

4. Tobar F. Acceso a los medicamentos en Argentina: diagnóstico y alternativas. Buenos Aires: Centro de Estudios de Estado y Sociedad; 2002. (Serie Seminarios Salud y Política Pública).

5. Sesma-Vázquez S, Pérez-Rico R, Sosa-Manzano CL, Gómez-Dantés O. Gastos catastróficos por motivos de salud en México: magnitud, distribución y determinantes. Salud Pública Méx 2005; 47 Suppl 1:S37-46.

6. Maceira D, Apella I, Barbieri E. Análisis del Programa REMEDIAR. Notas sobre evaluación y seguimiento. Washington DC: Banco Interamericano de Desarrollo; 2005.

7. Apella I. Acceso a medicamentos y producción pública: el caso Argentino. Buenos Aires: Centro de Estudios de Estado y Sociedad; 2006. (Documentos del CEDES 2006/26).

8. Whitehead M. The concepts and principles of equity and health. Int J Health Serv 1992; 22:429-45.

9. Whitehead M, Dahlgren G, Evans T. Equity and health sector reforms: can low-income countries escape the medical poverty trap? Lancet 2001; 358:833-6.

10. Wagstaff A, van Doorslaer E. Equity in health care finance and delivery. In: Culyerand AJ, Newhouse JP, editors. North Holland handbook of health economics. Oxford: Elsevier; 1998. p. 1803-62.

11. Wagstaff A. Poverty and health sector inequalities. Bull World Health Organ 2002; 80:97-105.
12. Maceira D. Financiamiento, aseguramiento y prestación de servicios de salud en Argentina. http:// danielmaceira.com.ar (accedido el Ene/2015).

13. Xu K, Evans DB, Kawabata K, Zeramdini R, Klavus J. Household catastrophic health expenditure: a multycountry anaylis. Lancet 2003; 362:111-7.

14. Torres AC, Knaul FM. Determinantes del gasto de bolsillo en salud e implicaciones para el aseguramiento universal en México: 1992-2000. In: Fundación Mexicana para la Salud, editor. Caleidoscopio de la salud. México DF: Fundación Mexicana para la Salud; 2003. p. 209-25.

15. Hanley GE, Morgan S, Hurley J, van Doorslaer E. Distributional consequences of the transition from age - based to income - based prescription drug coverage in British Columbia, Canada. Health Econ 2008; 17:1379-92.

16. Sojo A. Hacia la universalidad, con solidaridad y eficiencia: el financiamiento de la protección social en países pobres y desiguales. Santiago: Comisión Económica para América Latina; 2009. (Serie Seminarios y Conferencias, 55).

17. Apella I. Gasto de bolsillo en salud e impacto financiero sobre los adultos mayores en argentina. In: Anales de la Asociación Argentina de Economía Política, XLIV Reunión Anual. http://www.aaep.org. ar/anales/works/works2009/apella.pdf (accedido el Ene/2015).

18. Banco Mundial. Informe sobre el desarrollo mundial 1993. Invertir en salud. http://www-wds.world bank.org/ (accedido el Ene/2015).

19. Williamson J. Latin American adjustment: how much has happened? Washington DC: Institute for International Economics; 1990.

20. Hsiao WC. Abnormal economics in the health sector. Health Policy 1995; 32:125-39. 
21. Tobar F. Breve historia de la prestación de servicios de salud en Argentina. http://www.federicotobar. com.ar (accedido el Ene/2015).

22. Gogna M. Las reformas en el sector salud en Argentina y Chile: oportunidades y obstáculos para la promoción de la salud sexual y reproductiva. Buenos Aires: Centro de Estudios de Estado y Sociedad/Banco Interamericano de Desarrollo; 2004.

23. República Argentina. Ley n. 25.649 de 2002. Especialidades Medicinales. Ley de Promoción de la Utilización de Medicamentos por Nombre Genérico. http://www.infoleg.gob.ar/infolegInternet/ anexos/75000-79999/77881/norma.htm (accedido el Ene/2015).

24. Tobar F, Godoy Garraza L. Políticas para mejorar el acceso a los medicamentos. Notas desde el caso argentino. In: Rivas Vilchis JF, Molina Salazar RE, editores. Políticas farmacéuticas y estudios de utilización de medicamentos en Latinoamérica. México DF: Universidad Autónoma Metropolitana Itztapalapa; 2003. p. 27-40.

25. Ministério de la Saud. El programa REMEDIAR. Gestión y resultados de un modelo innovador en APS. Buenos Aires: Ministerio de Salud; 2006.

26. Maceira D. Financiamiento y equidad en el sistema de salud argentino. Buenos Aires: Centro de Estudios de Estados y Sociedad; 2002. (Serie Seminarios Salud y Política Pública).

27. Maceira D, Reynoso A. Gasto financieramente catastrófico y empobrecedor en salud. Argentina 1997-2005. http://danielmaceira.com.ar (accedido el Ene/2015).

28. Murray CH, Xu K, Klavus J, Kawabata K, Hanvoravongchai P, Zeramdini R, et al. Assessing the distribution of household financial contributions on the health system: concepts and empirical application. In: World Health Organization, editor. Health systems performance assessment: debates, methods and empiricism. Geneva: World Health Organization; 2003. p. 513-32.

29. Reyes FAV. Robustez a distribución para regresión cuantílica en datos longitudinales [Dissertación de Maestría]. Bogotá: Universidad Nacional de Colombia; 2011.

30. Sila IM. Regresión cuantiles [Dissertación de Maestría]. Santiago de Compostela: Universidade de Santiago de Compostela; 2010.

31. Johnson JB, Omland K. Model selection in ecology and evolution. Trends Ecol Evol 2004; 19:101-8.

32. Burnham K, Anderson D. Multimodel inference. Understanding AIC and BIC in model selection. Sociological Methods \& Research 2004; 33:261-304.

33. Fox J, Weisberg S. An $\{\mathrm{R}\}$ companion to applied regression. 2nd Ed. Thousand Oaks: Sage Publications; 2011.

34. Chatterjee BP. Regression analysis by example. $2^{\text {nd }}$ Ed. New York: Wiley; 2001.

35. Paradis E, Claude J, Strimmer K. APE: analyses of phylogenetics and evolution in R language. Bioinformatics 2004; 20:289-90.
36. Rangel TF, Diniz-Filho JAF, Bini LM. Towards an integrated computational tool for spatial analysis in macroecology and biogeography. Glob Ecol Biogeogr 2006; 15:321-7.

37. Stiglitz J. La economía del sector público. Barcelona: Editora A. Bosch; 2003.

38. República Argentina. Ley 26.688 de 2011. Salud pública. Investigación y producción pública de medicamentos, materias primas para la producción de medicamentos, vacunas y productos médicos. http://www.infoleg.gob.ar/infolegInter net/anexos/185000-189999/185041/norma.htm (accedido el Ene/2015).

39. Uthoff A. Alternativas para abordar los desafíos de la protección social en salud y pensiones en América Latina. Productividad, innovación, e inclusión social: lecciones de política de América Latina y el Este de Asia. Santiago: Corporación de Estudios para Latinoamérica; 2014.

40. Arrow K. Uncertainty and the welfare economics of medical care. The American Economic Review 1963; 53:941-73.

41. Falbo R, Vasallo C. Estructura de la oferta y política de medicamentos. In: Comisión Nacional Salud Investiga, editor. Política de medicamentos en la Argentina. Buenos Aires: Ministerio de Salud, Comisión Nacional Salud Investiga; 2007. p. 49-78.

42. Fundación ISALUD. El mercado de medicamentos en la Argentina. Serie Estudios de la Economía Real. http://www.isalud.org/documentacion/Tra bajodemedicamentos.pdf (accedido el Ene/2015).

43. Bisang R, Maceira D. Medicamentos: apuntes para una propuesta de política integral. Buenos Aires: LITTEC-Universidad Nacional General Sarmiento; 1999.

44. Homedes N, Ugalde A, Rovira Forns J. The World Bank, pharmaceutical policies and health reforms in Latin America. Int J Health Serv 2005; 35:691-717.

45. Tobar F, Sánchez D. El impacto de las políticas de medicamentos genéricos sobre el mercado de medicamentos en tres países del Mercosur. Montevideo: Fundación Carolina; 2005. (CeALCI 07/05).

46. Bloom D, Canning D, Sevilla J. The effect of health on economic growth: a production function approach. World Dev 2004; 32:1-13.

47. Sen A. ¿Por qué la equidad en salud? Rev Panam Salud Pública 2001; 11:302-9.

48. Instituto Nacional de Estadística y Censos. Censo Nacional de Población, Hogares y Viviendas, 2001. http://www.indec.gov.ar/ (accedido el Ene/2015).

49. Tobar F, Montiel L, Falbo R, Drake I. La red pública de atención primaria de salud en Argentina. http://www.federicotobar.com.ar/nf_pdf5/La Red_Publica.pdf (accedido el Ene/2015). 


\section{Abstract}

Medicines are an important part of household health spending. A progressive system for financing drugs is thus essential for an equitable health system. Some authors have proposed that the determinants of equity in drug financing are socioeconomic, demographic, and associated with public interventions, but little progress has been made in the empirical evaluation and quantification of their relative importance. The current study estimated quantile regressions at the provincial level in Argentina and found that old age (> 65 years), unemployment, the existence of a public pharmaceutical laboratory, treatment transfers, and a health system orientated to primary care were important predictors of progressive payment schemes. Low income, weak institutions, and insufficient infrastructure and services were associated with the most regressive social responses to health needs, thereby aggravating living conditions and limiting development opportunities.

National Drug Policy; Demography; Socioeconomic Factors

\section{Resumo}

Medicamentos são uma parte importante dos gastos em saúde do agregado familiar, portanto, ter um sistema progressivo de financiamento de medicamentos é fundamental para alcançar um sistema de saúde equitativo. Foi proposto que os determinantes da equidade no financiamento são fatores socioeconômicos, demográficos e ligados à intervenção pública, mas se avançou pouco em sua avaliação empírica e na quantificação de sua importância relativa. Neste trabalho, estimamos regressões por quantis no nível provincial na Argentina e encontramos que a população maior de 65 anos, o desemprego, a existência de laboratório de produção pública de medicamentos, transferências de tratamentos e orientação do sistema de saúde para atenção primária são importantes preditores da progressividade dos sistemas de pagamento. Baixa renda, instituições fracas, infraestrutura e oferta insuficiente de serviços resultam em mais regressivas respostas sociais às necessidades de saúde, agravando as condições de vida e limitando as oportunidades de desenvolvimento.

Política Nacional de Medicamentos; Demografia; Fatores Socioeconômicos
Recibido el 29/Ene/2015

Versión final presentada el 11/May/2015

Aprobado el 19/Jun/2015 